CERN-TH.7423/94

CTP-TAMU-59/94

ACT-17/94

hep-ph/9411281

\title{
The march towards no-scale supergravity
}

\author{
D.V. Nanopoulos \\ Center for Theoretical Physics, Department of Physics, Texas A\&M University \\ College Station, TX 77843-4242, USA \\ Astroparticle Physics Group, Houston Advanced Research Center (HARC) \\ The Mitchell Campus, The Woodlands, TX 77381, USA \\ CERN Theory Division, 1211 Geneva 23, Switzerland
}

\begin{abstract}
The different steps that led us to the discovery of no-scale supergravity are discussed from a very personal point of view. No-scale supergravity has been heralded as the most promising effective theory that describes physics below the Planck scale. In its string-derived form it holds the potential for a Dynamical Determination Of Everything (DDOE).
\end{abstract}

\section{Overview}

Among the many problems facing particle physics today, two fundamental ones pronouncedly stick out: (i) the origin of the different mass scales observed in Nature, and (ii) the miniscule upper bound on the cosmological constant. While, at first sight, these two problems seem to be unrelated, I will argue below that there is a deep-rooted correlation that suitably exploited may lead us to a natural and common resolution of these conundrums.

The success of the electroweak unification is based on the idea of Spontaneous Symmetry Breaking (SSB) of certain gauge symmetries, by allowing some scalar field $(\phi)$ to get a vacuum expectation value (v.e.v.), such that $\langle\phi\rangle=v \approx G_{F}^{-1 / 2}$, with $G_{F}$ the Fermi constant. The SSB is usually achieved by some judicious choice of the effective potential $V_{e f f}(\phi)$, completely arbitrary at this level of sophistication. All of the masses are then proportional to $v$, with the proportionality coefficient depending on the nature of the particle under consideration: gauge bosons $(W, Z): m_{W, Z} \approx g v$, with $g$ some electroweak gauge coupling; fermions: $m_{f_{i}} \approx h_{i} v$, with $h_{i}$ some arbitrary Yukawa coupling, and for the Higgs boson: $m_{\phi} \approx \lambda^{1 / 2} v$, with $\lambda$ some arbitrary quartic coupling. All sounds well, as we seem to have identified the origin of the different

\footnotetext{
${ }^{0}$ Invited talk presented at the International Conference on: "The History of Original Ideas and Basic Discoveries in Particle Physics", Erice, Italy, 29 July - 4 August 1994.
} 
mass scales, i.e., spontaneous breakdown $(\langle\phi\rangle \neq 0)$. This naive view turned out to be very deceptive. Each of the above masses $\left(m_{W, Z}, m_{f_{i}}, m_{\phi}\right)$ is the source of a cumbersome problem. To start with, who "orders" the Higgs potential to have the familiar "dumbbell" shape, so that $\langle\phi\rangle \neq 0$ ? Furthermore, as it is well-known, the quadratic ultraviolet divergences that plague scalar field theories shift the Higgs mass at the one-loop level by an amount

$$
\delta m_{\phi}^{2} \sim \Lambda^{2} \sim M_{P l}^{2}
$$

where $M_{P l}=\left(G_{N}\right)^{-1 / 2}$ is the natural cut-off of the theory, with $G_{N}$ Newton's gravitional constant, and assuming for simplicity that no intermediate mass scales are present. Clearly, a catastrophic result, the stability part of the gauge hierarchy problem. But even if we stabilized $m_{\phi}$, why is it that

$$
\left(\frac{G_{N}}{G_{F}}\right)^{1 / 2} \sim \frac{M_{W}}{M_{P l}} \sim 10^{-17},
$$

i.e., the magnitude part of the gauge hierarchy problem. In addition, a satisfactory resolution of the two-part gauge hierarchy problem will, in principle, leave untouched the fermion mass hierarchy problem, since

$$
h_{i}: \quad 10^{-6}(" e ") \longrightarrow \mathcal{O}(1)(" t ")
$$

a rather spread out fermion mass spectrum.

The SSB idea, while unavoidable for getting a renormalizable electroweak theory, consistent with all presently available experimental data, not only leaves us a bit unsatisfied about the "origin of mass", but it is also mainly responsible for the other fundamental problem, that of the cosmological constant $\left(\Lambda_{c}\right)$. A SSB vacuum would have a tremendously large energy density and this would give the physical vacuum an enormously large cosmological constant! It is fair to say that in todays popular theories, which extend the SSB ideas far beyond the electroweak scale, it is difficult to swallow the vast disparity between the "expected" $\mathcal{O}\left(M_{P l}^{4}\right)$ and the "observed" upper bound on the cosmological constant

$$
\frac{\Lambda_{c}}{M_{P l}^{4}} \leq \mathcal{O}\left(10^{-120}\right)
$$

This is the notorious cosmological constant problem, sometimes called, justifiably I think, the worst fine-tuning problem in the history of physics!

While SSB seems to be the common root of these two fundamental problems, we should not forget that both are related in a big way with gravity, which until now has been left out of our discussion. After all, another name for the mass would be gravitational charge, and the SSB vacuum energy metamorphises into the cosmological constant only when we couple our particle theory to gravity. We should then expect that the search for a possible common solution to these two problems has to involve 
gravity in a big way. This is exactly what happens in no-scale supergravity, which was discovered in $1983[1,2,3,4]$ in order to provide a common solution to the two fundamental problems, by exploiting their common origin and suggesting a very deep connection:

$$
\text { Existence of multitude of mass scales } \longleftrightarrow \text { Absence of cosmological constant }
$$

which may be considered as the "signature" of no-scale supergravity [1]-[6].

In the next sections I will provide my personal account of the different steps that brought us to the discovery of no-scale supergravity, and discuss its impact and its present status. This talk is by no means a complete review [7] of no-scale supergravity, neither of supersymmetry or supergravity, but some personal reminiscences of how things happened. Nevertheless, I do hope to convince the reader of why no-scale supergravity has been heralded by many [8], and not only by some of its inventors, as a very good candidate for an effective theory explaining physics below the Planck scale!

\section{Applied supergravity}

Among the many spectacular properties of supersymmetry (SUSY) or fermion-boson symmetry, the non-renormalization theorems play a fundamental role in solving the stability part of the gauge hierarchy problem [9]. Indeed, in an exactly supersymmetric world, the Higgs mass would receive no radiative corrections due to the fermionboson loop cancellation. But exact supersymmetry implies the equality between fermion and boson masses, not observed in Nature! In more realistic, broken SUSY theories, one replaces (1) by

$$
\delta m_{\phi}^{2}=\Lambda^{2}=m_{B}^{2}-m_{F}^{2} \lesssim \mathcal{O}\left(1 T e V^{2}\right)
$$

where the cut-off is now provided by the (mass) ${ }^{2}$ difference between the relevant bosons and fermions. If and only if it happens that these (mass) ${ }^{2}$ differences are smaller than $\mathcal{O}\left(1 \mathrm{TeV}^{2}\right)$, we would get a satisfactory resolution [9] of the stability part of the gauge hierarchy problem. Of course, in such case we effectively double the particle content of our theory, so that for each particle, its superpartner is available,

and with a mass not too far from the Fermi scale $\left(G_{F}^{-1 / 2}\right)$. The existence of an experimentally accessible superworld [10] is undoubtely the most dramatic consequence of invoking SUSY to resolve the stability part of the gauge hierarchy problem.

Thanks to the efforts of many people and many papers later [7], we realized that the notion of a realistic, spontaneously broken SUSY theory was in illusion. The notion of softly broken SUSY had to be introduced [11], which despite its arbitrariness and ad-hoc-ness seems to keep unscathed the elements of SUSY relevant to the solution of the stability problem [12]. Alas, the proliferation of the new arbitrary parameters that had to be introduced made the predictability of the theory mute. For every chiral multiplet, like say the electron $(\operatorname{spin} 1 / 2)$ - selectron $(\operatorname{spin} 0)$ one throws 
in some mass parameter $m_{0}$, ditto for the gauge multiplet, say the photon $(\operatorname{spin} 1)-$ photino (spin 1/2) one introduces a mass parameter $m_{1 / 2}$, while for the Yukawa couplings one has to introduce some SUSY breaking parameter $A[7,10]$. Furthermore, one has to introduce at least two Higgs doublets $\left(\phi_{t}, \phi_{b}\right)$, one coupled to the $Q=2 / 3$ quarks and the other to the $Q=-1 / 3$ quarks and $Q=-1$ leptons, thus introducing a new angle $\beta: \tan \beta \equiv\left\langle\phi_{t}\right\rangle /\left\langle\phi_{b}\right\rangle=v_{t} / v_{b}$, and a new mass parameter $\mu: \mu \phi_{t} \phi_{b}$, as well as its SUSY breaking counterpart $B[7,10]$. Counting the whole lot we arrive at 27 new parameters, not exactly a very desirable situation [10]. Actually, Low Energy Phenomenology (LEP) puts a lot of severe constraints on these parameters, that may be represented loosely as follows:

- Absence, at lowest order, of FCNC: "universal" $m_{0}[13]$.

- Existence of gauge coupling unification at super high energies, as "observed" at LEP [14]: "universal" $m_{1 / 2}$

- Absence of a substantial neutron electron dipole moment $d_{n} \leq \mathcal{O}\left(10^{-25} e-\mathrm{cm}\right)$ : "real" $A, B[15]$

- and of course stability: $m_{0}, m_{1 / 2}, \mu \lesssim \mathcal{O}(1 T e V)$

While all the above constraints can be met in the framework of softly broken SUSY theories, its arbitrariness and lack of dynamical reasoning makes one think that something better has to be invented. Indeed, until now we have considered only rigid or global SUSY, by tacitly ignoring gravity. While in the case of usual particle symmetries, the notion of global versus local is a matter of taste, in the case of SUSY theories the mere existence of gravity implies that SUSY theories are local. In other words, we have to address from the start locally supersymmetric theories or supergravity (SUGRA) theories [7]. In the case of locally supersymmetric theories, like in usual gauge theories, the only way to break them, at least in perturbation theory, is spontaneously.

One of the basic steps towards the construction of a realistic SUGRA model was taken by Arnowitt, Chamseddine, and Nath [16] and independently by Barbieri, Ferrara, and Savoy [17], when they proved that a spontaneously broken SUGRA theory reduces at low energies (with respect to the Planck scale $M_{P l}$ ) to a global SUSY theory plus some "soft breaking" terms! Exactly what "the doctor ordered"

$$
\mathcal{L}_{S U G R A} \stackrel{S S B}{-}-\stackrel{M_{P l}}{\longrightarrow} \mathcal{L}_{S U S Y} \oplus \text { "soft breaking" terms }
$$

where in this case all the soft breaking terms $\left(m_{0}, m_{1 / 2}, A, B\right)$ are functions of the "hidden sector" fields responsible for the SSB of supergravity

$$
\widetilde{m}=\widetilde{m}\left(\left\langle T_{i}\right\rangle\right) .
$$


In (9) I have generally represented the soft breaking parameters by $\widetilde{m}$ and $\left\langle T_{i}\right\rangle$ denote the hidden sector fields $T_{i}$ at their corresponding minima. Thus, in this new framework the soft breaking parametes may be viewed as boundary conditions, "given" by the vacuum expectation values of the corresponding functions at some energy scale close to $M_{P l}$. The gravitational origin of the soft breaking parameters, make the phenomenological constraints (7), demanding "universal" values, easier to satisfy. After all, as far as we know, gravity is blind to flavor, or $S U(2) \times U(1)$ or $S U(3)_{\text {color }}$ quantum numbers, so it makes a lot of sense to assume a common $m_{0}$ for all squarks and sleptons, and a common $m_{1 / 2}$ for all gauginos, as a boundary condition close, or at, the Planck scale. Of course, at lower energies these mass parameters, like all other mass parameters in Quantum Field Theory, are subject to renormalizations, due to controlable electroweak and strong interaction radiative corrections, as embodied in their corresponding renormalization group equations (RGEs) [7].

Before going any further, it is worth emphasizing that the search for a resolution of the stability part of the gauge hierarchy problem has led us to a practical use of SUSY theories, which until then they were kind of interesting field theories with some curious properties, like the non-renormalization theorems! Furthermore, it was found that for practical reasons, we had to include gravity, i.e., deal with supergravity theories, which we ought to have used anyway. It is fair to say that the resolution of the stability part of the gauge hierarchy problem has made SUSY/SUGRA theories not only mathematically elegant, but most importantly, perhaps, also physically relevant.

\section{Radiative Electroweak Symmetry Breaking (REWSB)}

One late afternoon, in the summer of ' 82 , John Ellis, Kyriakos Tamvakis, and myself were bouncing off ideas about how to use best the emerging framework of applied supergravity. The atmosphere was kind of grim, since we had noticed that the SUGRAtype "universality" of the soft breaking terms for squarks, sleptons, and Higgs bosons: $m_{0}^{2} \geq 0$, was bad news for phenomenology. The dilemma we were facing was the following: clearly $S U(3)_{\text {color }}$ and $U(1)_{e m}$ are unbroken symmetries and it is good that for squarks and sleptons $m_{0}^{2}>0$, i.e., no potential danger for spontaneous breakdown, where $m_{0}^{2}<0$ is needed. On the other hand, that is kind of bad for the electroweak interactions, where SSB entails $m_{0}^{2}<0$ ! What is going on? A grave problem. We decided to call it a day, and as I was walking back to my office it occurred to me that we had been very naive. We had forgotten about renormalization of these mass parameters! Supergravity just provides $m_{0}^{2} \geq 0$ at, or close to, the Planck scale, but then the different masses are renormalized differently, depending on the strong and electromagnetic "charges" of the corresponding particles.

It is basically the same type of idea that enabled Andrei Buras, John Ellis, Mary K. Gaillard, and myself [18], a few years before (in '77) to calculate successfully the $m_{b} / m_{\tau}$ ratio in GUTs and make a strong prediction for the number of generations: $N_{g}=3[18,19]$. Twelve years later this was successfully verified at LEP [20]. Again 
there, one started with a universal Yukawa coupling at the GUT scale $h_{b}=h_{\tau}$, and let mainly strong and electroweak radiative corrections to provide the "right" number at low energies $m_{b} / m_{\tau} \approx 3$.

I ran back to John's office and told them my thought, and I immediately knew we had it right, from the big happy smiles on their faces. They, on the spot, started writing some RGEs on the blackboard and we all got more and more excited. Very late in the evening John called me at home to assure me that he had looked at it again and he was convinced that it worked. Let us see how it works [21]. Consider the RGEs schematically [7]

$$
\frac{d \widetilde{m}^{2}}{d t}=\frac{1}{(4 \pi)^{2}}\left\{-\sum_{i=1,2,3} c_{i} g_{i}^{2} M_{i}^{2}+\alpha h_{t}^{2}\left(\sum_{k} \widetilde{m}_{k}^{2}\right)\right\}
$$

where $\widetilde{m}$ generically refers to squarks, sleptons, or Higgs bosons, $g_{1,2,3}$ are the $S U(3) \times$ $S U(2) \times U(1)$ gauge couplings, $M_{i}$ are the corresponding gaugino masses, $h_{t}$ is the top-quark Yukawa coupling (ignoring all other Yukawa couplings), and $c_{i}$ and $\alpha$ are numbers of order 1 , if not zero. It becomes clear now that as $t \equiv \ln Q^{2}$ decreases from the Planck scale down to the LEP scale, the different particle masses evolve differently because the $c_{i}$ and $\alpha$ depend on their particular $S U(3) \times S U(2) \times U(1)$ "charges". Here are the qualitative results of such an analysis [7].

- Sleptons: only $c_{1,2} \neq 0$ and thus, as we reach low energies, $m_{\tilde{l}}^{2}$ slightly increases from its $m_{0}^{2}>0$ value at Planck-like scales: $U(1)_{e m}$ is safe.

- Squarks: All $c_{1,2,3}, \alpha \neq 0$, but still, since $h_{t}^{2} \lesssim \mathcal{O}\left(g_{2}^{2}\right), m_{\tilde{q}}^{2}$ increases considerably as we reach low energies, thus always $m_{\tilde{q}}^{2}>0: S U(3)_{\text {color }}, U(1)_{\text {em }}$ are safe.

- Higgs: Here $c_{1,2}, \alpha \neq 0$ and there is a "fight" between the $-g_{2}^{2}$ term against the $+h_{t}^{2}$ term. Clearly, for big enough $h_{t}, h_{t}^{2} \approx \mathcal{O}\left(g_{2}^{2}\right)$, the exciting possibility emerges of decreasing $m_{\text {Higgs }}^{2}$, as we reach low energies, enough to hit zero or even negative values: $m_{h}^{2} \leq\left. 0\right|_{\text {low energy }}$, thus causing $\mathrm{SB}$ of the electroweak interactions! Because it is due to radiative corrections, i.e., of dynamical origin, we called it Radiative Electroweak Symmetry Breaking (REWSB).

Actually, detailed calculations showed the following highly interesting facts [7]:

1. In order to have REWSB, we needed a rather heavy top-quark $m_{t} \approx \mathcal{O}\left(M_{W}\right)$, a rather bold statement for ' 82 , where the top-mass lower bounds were in the $20 \mathrm{GeV}$ range!

2. We were able to determine dynamically the electroweak scale by basically determining the scale $\mu_{0}$ where $m_{h}^{2}$ hits zero:

$$
\frac{M_{W}}{M} \approx \frac{\mu_{0}}{M} \approx e^{-2 \pi / 3 \bar{\alpha}_{t}\left(1+\bar{A}_{t}^{2}\right)}
$$


where $M$ is some scale in the $\left(M_{G U T} \rightarrow M_{P l}\right)$ region, and $\bar{\alpha}_{t} \equiv \bar{h}_{t}^{2} / 4 \pi, \bar{A}_{t}$ are some "mean" values of $\alpha_{t}$ and $A_{t}$ in the $\left(M_{W} \rightarrow M\right)$ energy range. Of course, the similarity with the standard GUT relation

$$
\frac{\Lambda_{Q C D}}{M} \approx e^{-2 \pi \sin ^{2} \theta_{W} / 3 \alpha_{e m}}=e^{-2 \pi / 3 \alpha_{2}}
$$

is rather striking and certainly not accidental!

A few days after we finished the paper [21], I presented it at a meeting in Tokyo. After my talk, a shy Japanese fellow by the name of K. Inoue approached me and gave me a preprint. I did not look at it until I was airborne back to Geneva, and I had almost an apoplexy: they (K. Inoue, et. al. [22]) had done it also, and even in a more detailed and systematic way! Later on, many people [23] jumped on the band wagon and completed the picture, so that REWSB became the standard mechanism for SB of the electroweak interactions for a large part of our community. It will be unfair not to mention here some seeds of the REWSB idea due to Ibáñez and Ross [24]. They also relied on supersymmetry breaking to enforce $m_{h}^{2}<0$, but all their game was at the electroweak scale, i.e., they were not using any RGEs with boundary conditions at $M$, and only in the framework of global SUSY.

Clearly REWSB, through (11), provides a resolution of the magnitude part of the gauge hierarchy problem (2). Furthermore, the dynamical explanation of SB of electroweak interactions and at the same time the justification of why $S U(3)_{\text {color }} \times$ $U(1)_{e m}$ remains unbroken make REWSB an offer we cannot refuse. It certainly looks like a basic step towards the construction of a realistic SUGRA model. Nevertheless, there is a catch! We have tacitly assumed, as we run the RGEs (10), that the SUSY soft breaking terms $\left(m_{0}, m_{1 / 2}, \cdots\right)$ are not far above the Fermi or electroweak scale. Otherwise we have to "freeze" the running (10) at some scale $Q \gg M_{W}$, which may lead to either $S U(2) \times U(1)$ breaking at the "wrong" scale or no $S U(2) \times U(1)$ breaking at all! Catastrophic alternatives indeed! Then, someone may argue that we have tacitly replaced the magnitude part of the gauge hierarchy problem (2) by the SUSY breaking scale problem, i.e., why should

$$
\frac{\widetilde{m}}{M} \approx \mathcal{O}\left(10^{-16}\right)
$$

It looks like we have to overcome another stumbling block before we are able to successfully implement the REWSB. No-scale supergravity comes to its rescue.

\section{No-scale supergravity}

\subsection{No-scale Standard model}

The CERN Cafeteria is always a pretty busy place during lunch time, especially during summer time, when everyone is visiting CERN. A hot noon in the mid-summer of 
'83, I had lunch with Costas Kounnas in the CERN Cafeteria, when we saw Sergio Ferrara looking desperately for a table. We called him over and started discussing the talks we all had to deliver in a few days at the European Physical Society High Energy Physics Meeting in Brighton, England. Sergio was very complimentary on a paper we have just written [25] on the solution to the Higgs doublet-triplet splitting problem in supergravity, a rather notorious problem. I vividly remember my reaction to his comments. I told him that we were very happy with the resolution of this problem, but that were very unhappy with the extraneous fine-tuning that is needed in SUGRA theories so that they meet the cosmological constant upper bound (4). Sergio answered back by saying that at least we can do it in SUGRA, unlike SUSY theories where SUSY breaking implies positive cosmological constant! I told him that we needed to do better, something like a naturally flat potential that does not need fine-tuning, like the resolution of the Higgs doublet-triplet splitting problem that we all liked so much. He smiled enigmatically back at me and we left the discussion there. Very late in the afternoon he came to my office, closed the door and wrote something on the blackboard. Busy with preparing my transparencies, I paid no attention to him. Then he said, "why don't you check the potential in this case?" It took me a few seconds to check it and indeed it came out naturally flat, exactly what I had asked him. I was speechless, it was so simple and beautiful and we had not thought about this until that moment! Within the next two days we had the first draft of the paper [1], while we diminished our activity on our Brighton talks! Here is what we found [1]. In $N=1$ supergravity theories, the effective potential is given by (neglecting D-terms) $[7]$

$$
V_{S U G R A}=e^{G}\left[G_{i}\left(G^{-1}\right)_{j}^{i} G^{j}-3\right]
$$

where the Kähler function $G=G\left(T_{i}, T_{i}^{*}\right)$ is a real function of the fields $T_{i}$, and their complex conjugates $T_{i}^{*}$, and $G_{i}=\partial G / \partial T_{i} ; G^{j}=\partial G / \partial T_{j}^{*}$. Notice that the gravitino mass $m_{3 / 2}$, whose non-vanishing value at the ground state is a measure of SUGRA breaking, is given by

$$
m_{3 / 2}=e^{\langle G / 2\rangle}
$$

with $\langle G\rangle$ denoting the value of $G$ at the minimum of the potential (14). Clearly, one way to have $\Lambda_{c}=\left\langle V_{S U G R A}\right\rangle=0$ is to have $m_{3 / 2}=0$, i.e., unbroken supergravity, a phenomenologically unacceptable case. Another way is to arrange things so that

$$
\left\langle G_{i}\left(G^{-1}\right)_{j}^{i} G^{j}\right\rangle=3
$$

which demands an extraneous fine-tuning between different parameters, and thus highly improbable. Still another way, which is the mathematical expression of my question to Sergio is to demand

$$
G_{i}\left(G^{-1}\right)_{j}^{i} G^{j}=3
$$

as a field identity! That is to look for a specific function $G\left(T_{i}, T_{i}^{*}\right)$ that satisfies (17) for every value of $T_{i}$, thus getting a naturally vanishing (flat) potential, without 
necessarily a vanishing $m_{3 / 2}$, i.e., with SB supergravity! What Sergio had written on my blackboard was

$$
G=-3 \ln \left(T+T^{*}\right)
$$

which clearly obeys (17), in the simple case of one field, and thus leads to a flat $V_{S U G R A} \equiv 0$ according to (14). Actually, in the case of only one field, the solution (18) is the unique solution of (17)! Furthermore, at this classical level the mass of the gravitino remains undetermined, while non-zero

$$
m_{3 / 2}=\frac{1}{\left\langle\left(T+T^{*}\right)^{3 / 2}\right\rangle}
$$

since $\left\langle T+T^{*}\right\rangle$ is undetermined! So, here is what we have achieved [1]: a naturally vanishing cosmological constant, at least at the classical level, with SB supergravity, but with the SUGRA breaking scale $\left(\propto m_{3 / 2}\right)$ undetermined. Costas and I found this last part, undetermined SUGRA breaking at the classical level, very exciting, because we somehow foresaw some connection with our REWSB programme discussed in the previous section. Here things took a strange turn: Eugene Cremmer and Sergio Ferrara wouldn't even listen about possible connections to the REWSB mechanism, and we endep up [1] trying to un-flat the potential so that we got a fixed value of $m_{3 / 2}$ at the classical level! Despite all these needless excursions, Costas and I managed, under their noses, to sneak in some statements at the end of the paper about what we really believed was happening. Until this day I have not figured out why the CF part of our team (CFKN) [1] did not want to even listen about REWSB. Well, they were paving the way for John Ellis. John has never been accussed of not getting instantly an important physical point and true to his reputation, when he saw our (CFKN) paper [1], he also figured out that we had in our hands a mechanism for fixing dynamically all mass scales. He called from Munich, where he was at a summer school, and eagerly asked me not to write anything further before he was back at CERN! During this phone conversation the name no-scale supergravity was invented, as well as the specific generic scenario for dynamical determination of all mass scales. Again the idea is very simple. Applied supergravity usually provides us with some fixed arbitrary boundary conditions for the soft breaking parameters at, or close to, the Planck scale, assuming that all fields are at their minima, as shown in (9). In no-scale supergravity though, as depicted by (19), the soft breaking parameters are not fixed, at least not at the classical level, at, or close to, the Planck scale, thus replacing (9) by

$$
\widetilde{m}=\widetilde{m}\left(T_{i}\right)
$$

and thus we have to rely, once more, on radiative corrections to fix $\left\langle T_{i}\right\rangle$, and thus providing a dynamical determination of $\left(m_{0}, m_{1 / 2}, \cdots\right)$ ! Very similar to the way we determined the v.e.v. of the Higgs field, or equivalently $M_{W}$ (see (11)), in the standard model by the REWSB mechanism. But here we are facing a problem. The $T_{i}$ fields are usually singlets under $S U(3) \times S U(2) \times U(1)$, so how can we use strong and electroweak radiative corrections to fix their v.e.v.'s, a la the Higgs v.e.v.'s? Well, there is an extra small but profound step we have to take beyond the REWSB mechanism in order to 
complete our dynamical determination of the SUSY breaking scale. This extra step was taken by John Ellis, Thanasis Lahanas, Kyriakos Tamvakis, and myself about a month after the original CFKN paper [1], by constructing the first ever genuine realistic no-scale supergravity standard model [2]. Of course, in this realistic case the no-scale Kähler function (18) will have additional pieces representing the "observable" fields (quarks, Higgs, etc.), but they don't change at all our basic picture. The electroweak potential at the "observable" fields minimum, after the REWSB has occured, takes the generic form $[2,7]$

$$
\left(V_{E-W}\right)_{\text {min }}=-C m_{3 / 2}^{4}(T) \ln ^{2}\left(\frac{\kappa_{0} m_{3 / 2}^{2}(T)}{\mu_{0}^{2}}\right)
$$

where within $C$ we have absorbed all quantities that do not depend on $m_{3 / 2}, \kappa_{0}$ is a constant of order 1 , and $\mu_{0}$ is given by (11). Clearly, $m_{3 / 2}=m_{3 / 2}(T)$, as given by $(19)$, is an undetermined dynamical variable. Thus, we should minimize $\left(V_{E-W}\right)_{\min }$ further with respect to the field $T$ or equivalently with respect to $m_{3 / 2}(T)$, i.e., we are looking for the minimum minimorum of the electroweak potential

$$
\frac{d\left(V_{E-W}\right)_{\min }}{d m_{3 / 2}}=0
$$

which I have dubbed the no-scale condition. Applying this general no-scale condition (22) in the specfic case of $\left(V_{E-W}\right)_{\min }$, as given by (21), one determines dynamically $m_{3 / 2}$

$$
\frac{m_{3 / 2}^{2}}{\mu_{0}^{2}}=\frac{1}{\kappa_{0} e}=\mathcal{O}(1)
$$

and thus by (19), fixing dynamically $\left\langle T+T^{*}\right\rangle$.

This is the no-scale answer to the SUSY breaking scale problem (13). Thus, putting together (11) and (23) we get

$$
\frac{M_{W}}{M} \approx \frac{m_{3 / 2}}{M} \approx e^{\mathcal{O}\left(-1 / \alpha_{t}\right)}
$$

i.e., a dynamical determination of the fundamental scales of high-energy physics, the electroweak scale and the SUSY breaking scale. We also understand intuitively why they are close together: after all, in the no-scale framework, it is the physics of the electroweak potential that finally fixes $m_{3 / 2}$, thus one a priori expects $m_{3 / 2} \approx \mathcal{O}\left(M_{W}\right)$, what else is there?

Actually, there is more to our story. As originally observed in CFKN [1], and elaborated in great detail in EKN(I) [3], the flatness of the potential, when one used the no-scale Kähler form (18) is not just a caprice of the relevant equations, but it is due to some deep symmetry reasons. The flatness of the potential has its origin in the existence of a non-compact symmetry, $S U(1,1)$, which contains as subgroups, imaginary translations ( $T \rightarrow T+i \beta$ ), dilatations, and conformal transformations. We then discovered, to our delight, that the $S U(1,1)$ invariant "hidden" sector of our 
theory was isomorphic to the scalar sector of the $N=4$ extended supergravity. We could trace back the well-known properties of the absence of a scalar potential in the $\mathrm{N}=4$ theory, to its possession of the non-compact $S U(1,1)$ invariance. The absence of a scalar potential and the vanishing of the cosmological constant in higher $N>4$ (ungauged) supergravities are linked to their larger non-compact global invariance groups which contain $S U(1,1)$ as a subgroup. In other words, our choice of the noscale Kähler function (18) was not hard to justify as it had its dynamical origin in $N \geq 4$ supergravity which, at least at that time, were popular [26] for providing a fundamental theory of the Planck scale! An extra bonus, discussed in detail in EKN(I) [3], was the fact that, due to the $S U(1,1)$ non-compact symmetry, the observable lowmass fields have to be combined with the hidden sector in a non-trivial way if there is to be a non-trivial limit as $m_{3 / 2} / M_{P l} \rightarrow 0$. Light fields should have non-zero conformal weights if their superpotential terms are not to vanish when $m_{3 / 2} / M_{P l} \rightarrow$ 0 . The Yukawa couplings $\left(h_{i}\right)$ rescale differently, depending on their corresponding "conformal $(\subset S U(1,1))$ weights"

$$
\frac{m_{f_{i}}}{M_{W}} \approx \mathcal{O}\left(\frac{M_{W}}{M}\right)^{\lambda_{i}}, \quad i=1,2,3(\text { generation } \text { inde } x)
$$

thus providing the "seeds" for a resolution of the fermion mass hierarchy problem (3). For example, pick up $\lambda_{1} \approx 1 / 8, \lambda_{2} \approx 1 / 4, \lambda_{3} \approx 0$, rather normal values for the "weights", and we have the gross features of (3) explained. Of course, it remains to the fundamental theory, that would contain our no-scale structure, to fix these specific "weights" in a satisfactory way.

It is remarkable that the dynamical structure of the no-scale supergravity is such that not only solves satisfactorily both facets of the gauge hierarchy problem, as seen in (24), but it provides the "seeds" for resolving the fermion mass hierarchy problem, as seen in (25). The common root of the resolution of all the above problems lies closely in the flatness of the potential, i.e., a naturally vanishing cosmological constant, at least at the classical level, due to the existence of a dynamically derived (in $N \geq 4$ extended SUGRAs) non-compact, global $S U(1,1)$ symmetry. Thus, the deep correlation (5), which is the focal point of no-scale supergravity.

It should be stressed that in order for the no-scale programme to work, we have to assume $[3,7]$ that $\operatorname{Str} \mathcal{M}^{2}=\sum_{j}(-1)^{2 j}(2 j+1) \operatorname{Tr} \mathcal{M}_{j}^{2}$ vanishes. Otherwise there will be an extra term $\left(S \operatorname{St} \mathcal{M}^{2}\right) \Lambda^{2} \approx m_{3 / 2}^{2} M_{P l}^{2}$ in $V_{\text {eff }}$ that would imply either $m_{3 / 2}=0$ or $M_{P l}$ ! In other words, there would be no no-scale framework if $\operatorname{Str} \mathcal{M}^{2} \neq 0$. From the first days of no-scale models we stressed this point repeatedly (see e.g., a huge footnote on p. 408 in EKN(I) [3]), but without having an explicit fundamental theory at the Planck scale, we simply included the $\operatorname{Str} \mathcal{M}^{2}=0$ condition in the desiderata of the correct unified model. It turns out that this specific desideratum is a tough constraint to meet, at least in presently popular string models. 


\section{$4.2 \quad$ No-scale GUTs}

Costas Kounnas is usualy in an "excited state". In the winter of " $82-83$ he was almost "ionized". Toward the end of our work on the first EKN paper [3], he succeeded to generalize the no-scale Kähler potential (18) in a non-trivial way, for $N>1$ chiral fields, by utilizing $S U(N, 1)$ non-compact global symmetry instead of $S U(1,1)$. He also observed that in this case, one gets (8) but without the "soft-breaking" terms, i.e., $m_{0}=A=B=0$ ! For John and me that was the wrong way to go. Who needed unbroken global SUSY theories? Costas, charmed by the nice geometrical features of the $S U(N, 1)$ theory, wouldn't hear any criticism. He was talking continously about $S U(N, 1)$ to the extent that at any time I would see him in the corridor, I would hide in someone's office. During the Christmas/New Year holidays of '83-84, while I was in Athens, Greece, I started thinking about no-scale GUTs. Unfortunately, almost immediately I stopped on my "tracks" by very unpleasant thoughts. We had a rather severe problem to solve. In the general case when there is an intermediate scale in the theory, like $M_{G}$, the GUT scale usually taken to be $\mathcal{O}\left(10^{16} \mathrm{GeV}\right)\left(\ll M_{P l}\right)$, there is a contribution to the effective potential of the form

$$
\Delta V \approx\left(\left(M_{G}^{2}+m_{3 / 2}^{2}\right)^{2}-M_{G}^{4}\right) \ln \left(\frac{m_{3 / 2}^{2}}{M_{G}^{2}}\right) \approx \mathcal{O}\left(m_{3 / 2}^{2} M_{G}^{2}\right) \ln \left(\frac{m_{3 / 2}^{2}}{M_{G}^{2}}\right)
$$

which, by following the steps that led to (23), implies $m_{3 / 2} \approx \mathcal{O}\left(M_{G}\right)$, not a very desirable result! Clearly, we have to make sure that terms $\mathcal{O}\left(m_{3 / 2}^{2} M_{G}^{2}\right)$ do not appear in $\Delta V$, but how? Well, the only natural way that we know how to do this is to employ unbroken global SUSY for the heavy sector. And then it clicked. Suddenly, it occured to me that what Costas had found $\left(m_{0}=A=B=0\right)$ was the solution to our severe problem. Indeed, after my return to CERN, we worked out the basic framework that enabled us to construct no-scale GUTs [4]. Here are the main points. The no-scale Kähler potential for N-chiral fields (e.g., the hidden sector $T$-field and N-1 "observable" fields $\psi_{i}$ ) is given by [4] (neglecting the superpotential term for simplicity)

$$
G=-3 \ln \left(T+T^{*}-\sum_{i} \psi_{i} \psi_{i}^{*}\right)
$$

which has the following remarkable properties:

- Naturally vanishing cosmological constant, à la (17), due to the existence of an $S U(N, 1) \supset S U(1,1)$ non-compact global symmetry available in $N \geq 5$ extended supergravities [26].

- $m_{0}=A=B=0$, directly related to the existence of the $S U(N, 1)$ non-compact symmetry, thus averting the disastrous $\mathcal{O}\left(m_{3 / 2}^{2} M_{G}^{2}\right)$ heavy sector contribution to the effective potential.

On the other hand, we had at our disposal the possibility of turning on lowenergy sector gaugino masses $\left(m_{1 / 2} \neq 0\right)$, thus providing global SUSY breaking in 
the low-energy sector, while keeping the heavy sector supersymmetric. One has to be careful that radiative corrections do not filter into the heavy sector any SUSY breaking, and this is guaranteed if we push $M_{G} \approx \mathcal{O}\left(M_{P l}\right)$, and thus avoiding any renormalization of the heavy sector. We decided to go this way [4], and we relied on the future TOE to explain dynamically our assumptions:

- $m_{1 / 2} \neq 0$ and $M_{G} \approx \mathcal{O}\left(M_{P l}\right)$.

Actually, we were bold enough to enlarge the spectrum of the SUSY SU(5) by an extra $10+\overline{10}$, so that the usual case of $M_{G} \approx 10^{16} \mathrm{GeV}$ is replaced by $M_{G} \approx$ $\mathcal{O}\left(M_{P l}\right)$ ! A rather daring and provocative proposal in 1984 ! Clearly, the idea and its realization, of a GUT theory with $M_{G} \approx \mathcal{O}\left(M_{P l}\right)$ is not new and it has not been conceived only a posteriori in order to reconcile the LEP data indicating $M_{G} \approx$ $\mathcal{O}\left(10^{16} \mathrm{GeV}\right)$, under the assumption of minimal SUSY SU(5) spectrum, with the string scale $M_{S} \approx \mathcal{O}\left(10^{18} \mathrm{GeV}\right)$.

Before ending this section, just a few words about some important subsequent developments of no-scale GUTs that unfortunately I have no time or space to cover.

- We found that the condition $m_{3 / 2} \approx \mathcal{O}\left(M_{W}\right)$ could be abandoned and $m_{3 / 2}$ could be anything, e.g., $m_{3 / 2} \approx \mathcal{O}\left(M_{P l}\right)[5]$ or $m_{3 / 2} \leq \mathcal{O}(1 \mathrm{KeV})$ [6], as long as $m_{0}, m_{1 / 2} \approx \mathcal{O}\left(M_{W}\right)$. In certain cases there is a decoupling of $m_{3 / 2}$, and in everything I discussed above, its role is played by $m_{1 / 2}$. This is what we dubbed with Thanasis Lahanas, the "Gravitino Liberation Movement" (GLM) in our Physics Report review of no-scale supergravity [7], conceived in a liquid lunch in Ferney-Voltaire, a village in France, near CERN.

- With John Ellis and Kari Enqvist we entertained the very interesting possibility that a similar mechanism like the one that fixes $m_{3 / 2}=m_{3 / 2}(R e T)$, may be at work to fix dynamically $\theta_{Q C D}=0$. Indeed, we worked out examples [6] where $\theta_{Q C D}=\theta_{Q C D}(\operatorname{Im} T)$, while QCD nonperturbative dynamics provide a potential to the otherwise decoupled $I m T$ field, and thus ensuring that at its minimum $\theta_{Q C D}=0$, according to the well-known Peccei-Quinn theorem [27].

While clearly no-scale supergravity has some very appealing and unique properties, the big question remains: Is there any fundamental theory, at the Planck scale, that has no-scale supergravity as its infrared limit? The answer is emphatically yes, and the fundamental theory is string theory (ST).

\section{$5 \quad$ String no-scale supergravity}

In February of '85, and while a bunch of theorists including John Ellis and myself had lunch at the CERN cafeteria, we got some very exciting news. Gabriele Veneziano joined us, and as he was, literally, sitting down he broke the news "I just got a paper from Ed (Witten) that contains a derivation of no-scale supergravity from superstring 
theory". I still remember the Cheshire cat's smile on John's face, and my infinite impatience to finish lunch and get Ed's paper [28]. Using an "educated" dimensional reduction, highly imitative of compactification on a, then popular, Calabi-Yau manifold, Witten succeeded in deriving the following form for the Kähler function [28] (again neglecting, for simplicity, the superpotential term)

$$
G=-3 \ln \left(T+T^{*}-\sum_{i} \psi_{i} \psi_{i}^{*}\right)-\ln \left(S+S^{*}\right)
$$

which of course, is nothing else, but our no-scale GUTs Kähler function (27), amended by the inclusion of an extra no-scale-type term, corresponding to the dilaton $(S)$ field endemic in string theory. In this case the Kähler manifold is $\frac{S U(N, 1)}{S U(N) \times U(1)} \otimes \frac{S U(1,1)}{U(1)}$, strongly characteristic of the no-scale framework. Not even in my wildest dreams I would have thought that we would have a "derivation" of our no-scale framework within a year of its discovery, from a supposedly and purportedly fundamental theory, like string theory.

Actually, the derivation of an effective supergravity theory from string theory has become a special branch of research in string theory. It should be stressed that Witten's initial studies [28] have been replaced by much more sophisticated techniques, that have led to specific, exact forms of the Kähler function, always involving non-compact symmetries and always leading to some type or other of no-scale structure. The first complete studies in $D=4$ superstring theories were done [29] in the framework of the fermionic formulation (FFF) [30] and led to very interesting phenomenology. For the most recent study see Ref. [31], where all previous work is fairly completely mentioned.

The basic question that usually arises is, what is the physical reason that string theory provides a no-scale framework as its infrared limit? There is a simple reason for all this as follows:

(i) At the level of the space-time effective action, we shouldn't forget that we start with something like $N=1$ SUGRA in $D=10$, which can be seen as $N=4$ SUGRA in $D=4$, and thus our space-time effective action should, at some level, contain among its symmetries the non-compact symmetries characteristic of extended supergravities, and as discussed in the previous section, the raison d'etre of the no-scale structure. Note that in the case of (FFF) [30], we always start with $N=4$ SUGRA in $D=4$, thus the above comments cover also this case.

(ii) At the level of the 2-d world-sheet action, we shouldn't forget that conformal invariance entails the vanishing of the 2 -d world-sheet $\beta$-functions $\beta_{\phi}$, which in turn provide the Equations of Motion (EOM) for the $D=4$ physical fields $\phi$

$$
\beta_{\phi}=\frac{d V_{e f f}}{d \phi}=0
$$

For certain fields, called moduli (like the $T$ and $S$ discussed above), the vanishing of the $\beta$-functions (or $d V_{e f f} / d \phi$ ) does not occur only at a critical point, but at a 
critical line, thus providing flat directions for these fields, at least in perturbation theory

$$
\left.\frac{d V_{e f f}}{d \phi}\right|_{\phi \in \text { critical line }}=0
$$

But, flat directions are the logo of the no-scale framework, and we found here that they have their roots deep into the basics of string theory!

Of course, one expects much more sophisticated no-scale Kähler structures [31], that we envisioned initially [3, 4], since here there is a multitude of moduli fields and after all, in string theory the no-scale structure is dynamically derived, it is not intuitively imposed. You get, what you get! Actually, this is good news because now we can address dynamically some of the assumptions that we have made before, including the vanishing of $S \operatorname{tr} \mathcal{M}^{2}$, and the need for $m_{0}=A=B=0 ; m_{1 / 2} \neq 0$ and $M_{G} \approx \mathcal{O}\left(M_{P l}\right)$ in the case of no-scale GUTs. Indeed, there is a very simple, "realistic" example that shows how one can avert the $\operatorname{Str} \mathcal{M}^{2} \neq 0$ problem. Consider the case of 3 -singlets (moduli) fields $T, S, U$ and $N-1$ "charged" fields $\psi_{i}$, entering the Kähler function $G$ in the following way [32] (including the superpotential term $W)$

$$
G=-3 \ln \left(T+T^{*}-\sum_{i} \psi_{i} \psi_{i}^{*}\right)-\ln \left(S+S^{*}\right)-\ln \left(U+U^{*}\right)+\ln |W(\psi, S, U)|^{2}
$$

corresponding to a stringy-inspired Kähler manifold $\frac{S U(N, 1)}{S U(N) \times U(1)} \times \frac{S U(1,1)}{U(1)} \times \frac{S U(1,1)}{U(1)}$. Clearly this looks very familiar (see (27),(28)) and the extra field $U$ makes nothing to change the good features mentioned above. On the other hand, it is tremendously helpful in cancelling the $\operatorname{Str} \mathcal{M}^{2}$ term. Indeed, using the techniques developed in EKN(II) [4] one easily finds [32]

$$
\operatorname{Str} \mathcal{M}^{2} \propto(N+2)-1-\left(\frac{(N+1)}{3} G_{T} G^{T}\right)=0
$$

since $G_{T} G^{T}=3$ from the vacuum energy cancellation. Thus, our basic assumption of $\operatorname{Str} \mathcal{M}^{2}=0$ can be met in realistic string-inspired/derived no-scale models. It shouldn't escape our notice the deep connection between the vanishing of Str $\mathcal{M}^{2}$ and the vanishing of the cosmological constant. Concerning the highly desirable boundary conditions $m_{0}=A=B=0 ; m_{1 / 2} \neq 0$, they are endemic in string theories, and all kinds of different approximations usually yield the above no-scale boundary conditions. Concerning the $M_{G} \approx \mathcal{O}\left(M_{P l}\right)$, it is enough to remind ourselves that string theory provides "free" gauge coupling unification at a dynamically determined scale $M_{\text {string }} \approx \mathcal{O}\left(6 \times 10^{17} \mathrm{GeV}\right)$ thus making the identification $M_{G} \approx \mathcal{O}\left(M_{\text {string }}\right)$ rather natural. It is left upon to some extra representations, amending the minimal SUSY Standard Model, to push the apparent LEP unification scale of $\mathcal{O}\left(10^{16} \mathrm{GeV}\right)$ close to the string scale, $M_{\text {string }} \approx \mathcal{O}\left(6 \times 10^{17} \mathrm{GeV}\right)$, exactly as envisaged in EKN(II) [4] more than ten years ago. 
It is very encouraging that our basic assumptions in building the no-scale framework may find a natural explanation in string theory. Furthermore, it is easy to see that in string no-scale supergravity we have the potential for calculating basically from first principles dynamically all relevant parameters. Here are the basic steps:

- $g^{2}=1 /\langle\operatorname{Re} S\rangle$, the string unification gauge coupling is given in terms of $\langle\operatorname{Re} S\rangle$, introduced in (28), that is expected to be fixed dynamically, presumably nonperturbatively, but still one naturally expects $\langle\operatorname{Re} S\rangle / M_{\text {string }} \approx \mathcal{O}(1)$, thus $g^{2} \approx$ $\mathcal{O}(1)$ as indicated phenomenologically. Notice that once we have determined dynamically the value of $g^{2}(\approx \mathcal{O}(1))$ and the string scale $M_{\text {string }} \approx \mathcal{O}(6 \times$ $\left.10^{17} \mathrm{GeV}\right)$, we can determine dynamically the values of $\alpha_{3}, \alpha_{2}$, and $\alpha_{e m}$ at the LEP scale!

- $\left.h_{i}\right|_{Q \approx M_{\text {string }}} \approx g\left(\langle\phi\rangle / M_{\text {string }}\right)^{n}$, the Yukawa couplings are given in terms of $g$, and some combination of VEVs of appropriate singlets [33]. Clearly, $\langle\phi\rangle / M_{\text {string }} \approx$ $\mathcal{O}(1 / 10)$, as indicated in many specific string models, and $n=0,1,2$, corresponding to $3 \mathrm{rd}, 2 \mathrm{nd}$, and 1 st generation respectively, "reproduces" with sufficient accuracy the observed fermion mass spectrum [34]. Note that $h_{t} \approx \mathcal{O}(g)$, as observed [35] and as required by REWSB mechanism. As an example of this programme, let me mention a rather amusing, interesting, and novel relation that we got with Jorge Lopez back in 1990 [36]

$$
\frac{m_{c}}{m_{t}} \sim \frac{1}{2}\left(\frac{m_{e}}{m_{\tau}}\right)^{1 / 2}
$$

which implies a top-quark mass $\mathcal{O}(160-170 \mathrm{GeV})$ ! Incidentally, detailed studies [37], by Jorge Lopez, Nino Zichichi, and myself, of the top-quark Yukawa coupling in string theory $\left(h_{t} \approx \mathcal{O}(g)\right)$, enabled us to make a firm prediction of $m_{t} \in[150-180 \mathrm{GeV}]$ range, just a few months before the offical FNAL/CDF announcement $[35]$.

- $\widetilde{m} / M_{\text {string }} \approx \frac{m_{W}}{M_{\text {string }}} \sim \mathcal{O}\left(e^{-1 / \alpha_{t}}\right)$, by employing the no-scale mechanism with a "heavy" top quark dynamically derived in the previous step.

Thus, string no-scale supergravity may provide us with a framework where basically all physically relevant quantities/parameters would be dynamically determined, i.e., a Theory of Everything (TOE) with the ability of Dynamical Determination Of Everything (DDOE). Nevertheless, we shouldn't get carried away by the phenomenal success of the good "mix" of string theory and the no-scale mechanism. Even, if, which I personally doubt, it turns out that, string theory was an ephimeral illusion, the no-scale supergravity will be always there, available as an effective theory, able to give a good description of reality at energies below the Planck scale.

Until now I have deliberately avoided any discussion on the value of the cosmological constant $\left(\Lambda_{c}\right)$ beyond the classical level. Here, I will try to explain my attitude and try to answer some skepticism that might have been born concerning the physics 
of the no-scale models. We started by demanding naturally a zero tree-level cosmological constant and this was related to the $S U(1,1)$ non-compact symmetry, but then with the introduction of the observable sector the vacuum energy becomes non-zero $V_{E-W}=-\mathcal{O}\left(M_{W}^{4}\right) \neq 0(21)$. This results in a cosmological constant $\Lambda_{c}=10^{-60} M_{P l}^{4}$, which is much greater than the one actually observed (4) but still very small in comparison with an energy whose natural order is expected to be $\mathcal{O}\left(m_{3 / 2}^{2} M_{P l}^{2}\right)$. At this point, one should not forget that we were dealing with an effective low-energy theory. In other words, we considered the tree-level gravitational interactions and then took the flat limit $M_{P l} \rightarrow \infty$. In this way, at low energy one completely forgets the gravitational interactions and considers only the electroweak and strong forces. One cannot expect to find vanishing contributions to the cosmological constant from only the radiative corrections of the non-gravitational interactions. The vanishing of the cosmological constant is a matter that concerns the gravitational interactions and these were ignored at scales $\mu \ll M_{P l}$ by letting $M_{P l} \rightarrow \infty$ (flat limit). The full theory should give $\Lambda_{c}<10^{-120} M_{P l}^{4}$ when gravitational corrections are taken into account, but at present we do not know how to do that. Hopefully, string no-scale supergravity will succesfully resolve this problem.

During the last few years, with my close collaborators Jorge Lopez and Nino Zichichi, both firm believers and strong practitioners of no-scale supergravity, we have developed a framework of studying string no-scale supergravity from its lofty string origin down to the nitty-gritty details of its experimental consequences [38]. Only then, we believe, we can have the complete theory and we will be able to "prove" or refute it. We believe that we are not far from developing a genuine, experimentally accessible, no-parameter stringy no-scale model that will be an explicit realization of the basic steps I sketched above. If we are lucky enough, maybe someone of us will give you the details of how it happened, at the next History conference of this series.

\section{Acknowledgments}

It is a great pleasure for me to express my sincere thanks to the co-discoverers of the no-scale supergravity (in alphabetical order): E. Cremmer, J. Ellis, S. Ferrara, C. Kounnas, A. Lahanas, and K. Tamvakis for the great, exciting, and somehow singularly unique times that we shared together. Many thanks also are due to my close collaborators Jorge Lopez and Nino Zichichi for not only keeping the focus and the spirit of no-scale supergravity very well alive, but also for bringing it to new heights, by digging deeper and deeper into its structure. Finally, I would like to express my deep gratitude to Harvey Newman and Tom Ypsilantis, for an impecably organized conference and for giving me the opportunity to address such an exceptionally talented audience, including lots of heroes of my youth. This work has been supported in part by DOE grant DE-FG05-91-ER-40633. 


\section{References}

[1] E. Cremmer, S. Ferrara, C. Kounnas, and D. V. Nanopoulos, Phys. Lett. B 133 (1983) 61.

[2] J. Ellis, A. Lahanas, D. V. Nanopoulos, and K. Tamvakis, Phys. Lett. B 134 (1984) 429 .

[3] J. Ellis, C. Kounnas, and D. V. Nanopoulos, Nucl. Phys. B 241 (1984) 406.

[4] J. Ellis, C. Kounnas, and D. V. Nanopoulos, Nucl. Phys. B 247 (1984) 373.

[5] J. Ellis, C. Kounnas, and D. V. Nanopoulos, Phys. Lett. B 143 (1984) 410.

[6] J. Ellis, K. Enqvist, and D. V. Nanopoulos, Phys. Lett. B 147 (1984) 99 and Phys. Lett. B 151 (1985) 357.

[7] For a review see A. B. Lahanas and D. V. Nanopoulos, Phys. Rep. 145 (1987) 1.

[8] See e.g., J. Burton, M. K. Gaillard, and V. Jain, Phys. Rev. D 41 (1990) 3118.

[9] E. Witten, Nucl. Phys. B 188 (1981) 513; L. Maiani, Proc. Summer School on Particle Physics, Gif-sur-Yvette, 1979 (IN2P3, Paris, 1980) p. 3; R. K. Kaul, Phys. Lett. B 109 (1982) 19.

[10] For a review see, Status of the Superworld, from theory to experiment, J. L. Lopez, D. V. Nanopoulos, and A. Zichichi, Prog. Part. Nucl. Phys. 33 (1994) $303-395$.

[11] L. Girardello and M. T. Grisaru, Nucl. Phys. B 194 (1982) 65.

[12] S. Dimopoulos and H. Georgi, Nucl. Phys. B 193 (1981) 150; N. Sakai, Z. Phys. C11 (1982) 153.

[13] J. Ellis and D. V. Nanopoulos, Phys. Lett. B 110 (1982) 44.

[14] J. Ellis, S. Kelley, and D. V. Nanopoulos, Phys. Lett. B 249 (1990) 441; see Ref. [10] for a full account of this exciting development and references therein.

[15] R. Arnowitt, J. L. Lopez, and D. V. Nanopoulos, Phys. Rev. D 42 (1990) 2423, and references therein.

[16] R. Arnowitt, A. H. Chamseddine, and P. Nath, Phys. Rev. Lett. 49 (1982) 970.

[17] R. Barbieri, S. Ferrara, and C. Savoy, Phys. Lett. B 119 (1982) 343.

[18] A. Buras, J. Ellis, M. K. Gaillard, and D. V. Nanopoulos, Nucl. Phys. B 135 (1978) 66 . 
[19] D. V. Nanopoulos and D. A. Ross, Nucl. Phys. B 157 (1979) 273, Phys. Lett. B 108 (1982) 351, and Phys. Lett. B 118 (1982) 99.

[20] L3 Collab., B. Adeva, et. al., Phys. Lett. B 231 (1989) 509; ALEPH Collab., D. Decamp, et. al., Phys. Lett. B 231 (1989) 519; OPAL Collab., M. Akrawy, et. al., Phys. Lett. B 231 (1989) 530; DELPHI Collab., P. Aarnio, et. al., Phys. Lett. B 231 (1989) 539.

[21] J. Ellis, D. V. Nanopoulos, and K. Tamvakis, Phys. Lett. B 121 (1983) 123.

[22] K. Inoue, A. Kakuto, H. Komatsu, and S. Takeshita, Prog. Theor. Phys. 67 (1982) 1889 and 68 (1982) 927.

[23] J. Ellis, J. Hagelin, D. V. Nanopoulos, and K. Tamvakis, Phys. Lett. B 125 (1983) 275; L. Alvarez-Gaumé, J. Polchinski, and M. Wise, Nucl. Phys. B 221 (1983) 495; L. Ibañéz and C. López, Phys. Lett. B 126 (1983) 54 and Nucl. Phys. B 233 (1984) 545; C. Kounnas, A. Lahanas, D. V. Nanopoulos, and M. Quirós, Phys. Lett. B 132 (1983) 95 and Nucl. Phys. B 236 (1984) 438; K. Inoue, A. Kakuto, H. Komatsu, and S. Takeshita, Prog. Theor. Phys. 68 (1982) 927.

[24] L. Ibáñez and G. Ross, Phys. Lett. B 110 (1982) 215.

[25] C. Kounnas, D. V. Nanopoulos, M. Srednicki, and M. Quiros, Phys. Lett. B 127 (1983) 82.

[26] E. Cremmer and B. Julia, Phys. Lett. B 80 (1978) 48, Nucl. Phys. B 159 (1979) 141; J. Ellis, M. K. Gaillard, and B. Zumino, Phys. Lett. B 94 (1980) 343.

[27] R. Peccei and H. Quinn, Phys. Rev. Lett. 38 (1977) 1440, Phys. Rev. D 16 (1977) 1791.

[28] E. Witten, Phys. Lett. B 155 (1985) 151.

[29] I. Antoniadis, J. Ellis, E. Floratos, D. V. Nanopoulos, and T. Tomaras, Phys. Lett. B 191 (1987) 96; S. Ferrara, L. Girardello, C. Kounnas, and M. Porrati, Phys. Lett. B 193 (1987) 368.

[30] I. Antoniadis, C. Bachas, and C. Kounnas, Nucl. Phys. B 289 (1987) 87; I. Antoniadis and C. Bachas, Nucl. Phys. B 298 (1988) 586; H. Kawai, D.C. Lewellen, and S.H.-H. Tye, Phys. Rev. Lett. 57 (1986) 1832; Phys. Rev. D 34 (1986) 3794; Nucl. Phys. B 288 (1987) 1; R. Bluhm, L. Dolan, and P. Goddard, Nucl. Phys. B 309 (1988) 330; H. Dreiner, J. L. Lopez, D. V. Nanopoulos, and D. Reiss, Nucl. Phys. B 320 (1989) 401.

[31] J. L. Lopez, D. V. Nanopoulos, and K. Yuan, Phys. Rev. D 50 (1994) 4060.

[32] S. Ferrara, C. Kounnas, and F. Zwirner, CERN-TH.7192/94 (hep-th/9405188). 
[33] S. Kalara, J. L. Lopez, and D. V. Nanopoulos, Nucl. Phys. B 353 (1991) 650.

[34] J. L. Lopez and D. V. Nanopoulos, Nucl. Phys. B 338 (1990) 73, Phys. Lett. B 251 (1990) 73, Phys. Lett. B 256 (1991) 150.

[35] CDF Collaboration, F. Abe, et. al., Phys. Rev. Lett. 73 (1994) 225 and Phys. Rev. D 50 (1994) 2966.

[36] J. L. Lopez and D. V. Nanopoulos, Phys. Lett. B 268 (1991) 359.

[37] J. L. Lopez, D. V. Nanopoulos, and A. Zichichi, Phys. Lett. B 327 (1994) 279.

[38] For a review see: $S U(5) \times U(1):$ A string paradigm of a TOE and its experimental consequences, J. L. Lopez, D. V. Nanopoulos, and A. Zichichi, in From Superstrings to Supergravity, Proceedings of the INFN Eloisatron Project 26th Workshop, edited by M. J. Duff, S. Ferrara, and R. R. Khuri (World Scientific, Singapore 1993), p. 284; Rivista del Nuovo Cimento, 17(1994) 1; Ref. [10]. 